\title{
High-Yield Expression and Purification of Recombinant $\sigma B$ Protein of Avian Reovirus (ARV) in Prokaryotic System
}

\author{
Sanjeevna K. Minhas ${ }^{1 *}$, Nitin M. Kamble ${ }^{1}$, J.M. Kataria ${ }^{2}$, \\ C. Madhan Mohan ${ }^{1}$ and Sohini Dey ${ }^{1}$ \\ ${ }^{1}$ Recombinant-DNA Laboratory, Division of Veterinary Biotechnology, Indian Veterinary \\ Research Institute, Izatnagar, UP - 243122, India \\ ${ }^{2}$ Central Avian Research Institute, Izatnagar, UP - 243122, India \\ *Corresponding author
}

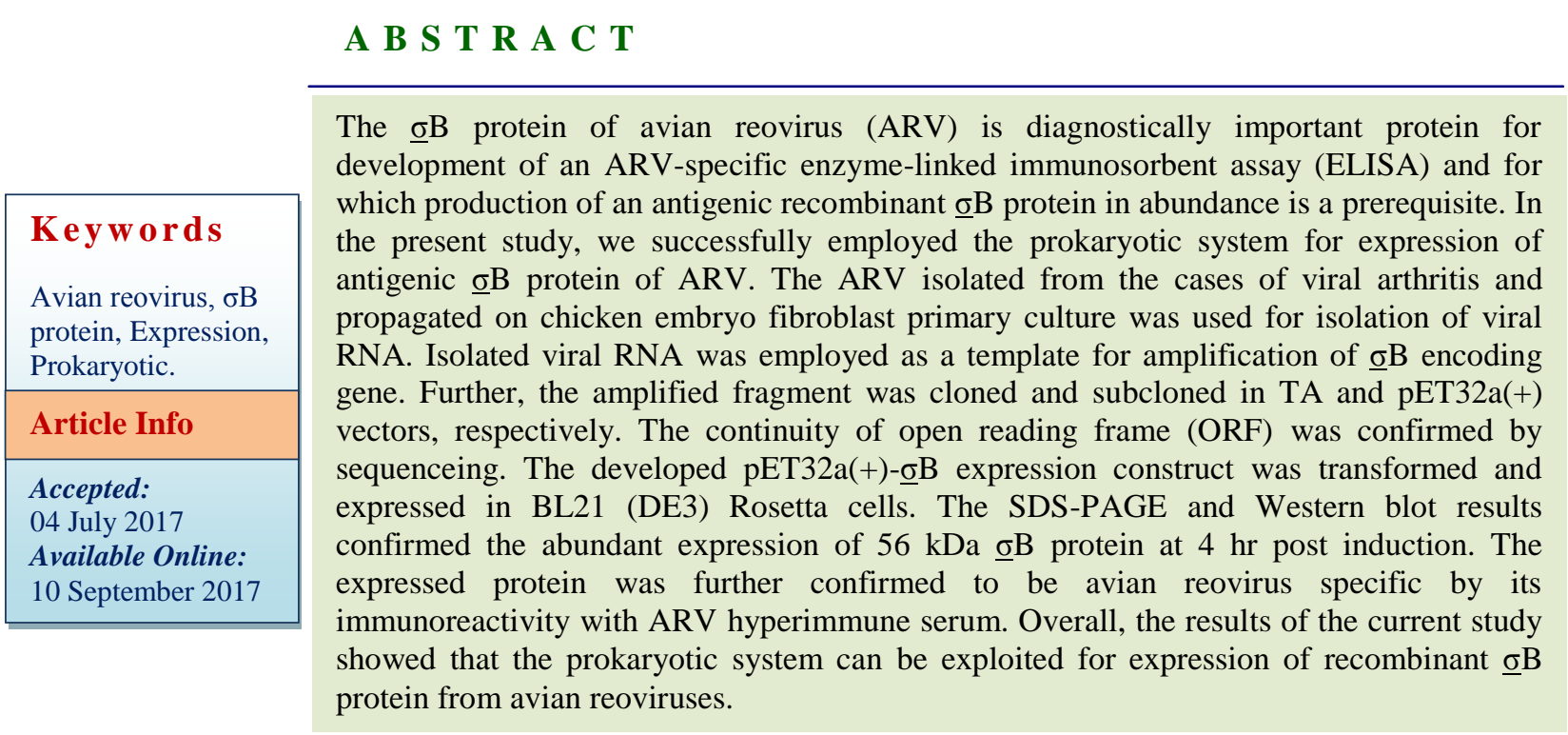

\section{Introduction}

Avian reovirus (ARV) infection is a ubiquitous disease of poultry, affecting mostly broiler breeds. Pathogenic effects of ARV such as arthritis and tenosynovitis are attributed to significant economic losses to poultry industry. ARV is classified under genus Orthoreovirus of family Reoviridae (Van Regenmortel et al., 2000). The genome of ARV encodes at least ten structural proteins $(\lambda \mathrm{A}, \lambda \mathrm{B}, \lambda \mathrm{C}, \mu \mathrm{A}, \mu \mathrm{B}, \mu \mathrm{BC}, \mu \mathrm{BN}, \underline{\sigma} \mathrm{C}$, $\underline{\sigma} \mathrm{A}$ and $\underline{\sigma} \mathrm{B})$ and four non-structural proteins
( $\mu$ NS, P10, P17 and $\sigma \mathrm{NS}$ ) (Benavente and Martínez-Costas, 2007). Among these proteins, S3 gene-encoded $\underline{\sigma} \mathrm{B}$ is a major outer capsid protein of $A R V$, functioning in the induction of cell fusion and viral pathogenesis (Ni and Ramig, 1993). ARV $\underline{\sigma} B$ protein is structurally similar to the $\underline{\sigma} \mathrm{B}$ protein of Duck reoviruses and Mammalian reoviruses (Vakharia et al., 1996; Yin et al., 1997) and share functional similarity with them. The $\underline{\sigma} \mathrm{B}$ protein is also known to induce group-specific 
neutralizing antibodies against ARV and can be used for detection of ARV-specific antibodies in infected or vaccinated chickens.

Timely and specific diagnosis of ARV outbreak is a prerequisite for the implementation of vaccination programs for efficient control and subsequent prevention of outbreaks.

Various serological diagnostic techniques have been developed for efficient detection of antibodies against ARV, including serum neutralization test (Wickramasinghe et al., 1993), AGID (Meanger et al., 1995), immunoblot assay (Ide and Dewitt, 1979) and ELISA (Liu et al., 2002). Several indirect ELISA diagnostic test based on recombinant $\underline{\sigma} \mathrm{C}$ and $\underline{\sigma} \mathrm{B}$ protein have been developed and validated for detection of antibodies against ARV (Zhang et al., 2007).

Extensive sequence similarity of recombinant $\underline{\sigma} \mathrm{B}$ proteins with isolates prevalent in local outbreaks is a prerequisite for more specific and accurate detection of ARV infection. Previous reports of sequencing and phylogenetic analysis of $\underline{\sigma} \mathrm{B}$ encoding gene of Indian ARV isolate has revealed $89.9 \%$ sequence homology with ARV isolates from other countries (Kumari S. et al., 2011). The substantial sequence divergence may contribute to significant reduction in the specificity and accuracy of ELISA-based detection.

Therefore, expression and purification of recombinant $\underline{\sigma} B$ protein from Indian $A R V$ isolate is favorable for the development of specific and accurate ELISA test for timely detection of ARV outbreaks in India.

In this study, we studied cloning, expression and purification of the $\underline{\sigma} \mathrm{B}$ protein of an Indian ARV isolate in $E$. coli based prokaryotic expression system.

\section{Materials and Methods}

\section{Cells and virus}

The ARV isolate used in the current study was maintained in the Avian Diseases Section, Division of Pathology, IVRI, Izatnagar (Bareilly, India). The virus was isolated from chicken flocks with the history of viral arthritis and malabsorption syndrome from a poultry farm in India (Kataria et al., 1982). The virus was adapted and subsequently maintained in chicken embryo fibroblast (CEF) cell culture by repeated passaging. The CEF were cultured in Glasgow minimal essential medium (GMEM) (Sigma, USA) containing $10 \%$ foetal calf serum (FCS) (Thermo Scientific, USA), 100 $\mathrm{U} / \mathrm{mL}$ penicillin and $100 \mu \mathrm{g} / \mathrm{mL}$ streptomycin at $37{ }^{\circ} \mathrm{C}$ with $5 \% \mathrm{CO}_{2}$ level. The supernatant of CEF culture positive for ARV virus was used as viral inoculum to infect a monolayer (70-80\% confluency) of CEF cells. After ARV inoculation, the CEF cells were washed and incubated in GMEM containing $2 \%$ FCS. After the observation of visible cytopathic effects typical of ARV, CEF culture was harvested by three freeze-thaw cycles and clarified by centrifugation at $10,000 \times \mathrm{g}$ for 10 min in an $\mathrm{F} 2402 \mathrm{H}$ rotor (Beckman, USA).

\section{RNA isolation, cDNA preparation and cloning}

Total viral RNA was isolated from ARVinfected CEF cells using TRIzol ${ }^{\circledR}$ reagent (Invitrogen, Carlsbad, CA), as per the manufacturers protocol. The extracted RNA was reverse-transcribed to synthesize the first strand cDNA using gene specific primers and Thermoscript ${ }^{\mathrm{TM}}$ RT-PCR kit (Invitrogen, Carlsbad, USA). Synthesized cDNA was further used as a template for amplification and TA cloning of $\underline{\sigma} \mathrm{B}$ encoding gene in pTZ57R/T vector. Full length $\underline{\sigma} \mathrm{B}$ gene sequence was amplified from cDNA using the 
following primer pairs (forward 5'CCCGGATCCATGGAGGTACGTGTGCCA $\mathrm{AA}-3$, and reverse 5'CCCAAGCTTTTACCAACCACACTCCAC AAC-3'). BamHI and HindIII restriction sites placed in the forward and reverse primers, respectively, were used to facilitate cloning. PCR amplification was carried out using the following cycling conditions: $94{ }^{\circ} \mathrm{C}$ for $5 \mathrm{~min}$ followed by 30 cycles of $94{ }^{\circ} \mathrm{C}$ for $1 \mathrm{~min}, 55$ ${ }^{\circ} \mathrm{C}$ for $1 \mathrm{~min}, 72{ }^{\circ} \mathrm{C}$ for $1.5 \mathrm{~min}$, with a final extension of $72{ }^{\circ} \mathrm{C}$ for $10 \mathrm{~min}$. The PCR amplified gene product was cloned into a T/A cloning vector $\mathrm{pTZ57R/T}$ (MBI Fermentas, Germany). A band size of 1104bp ( 1.1 Kbp) was observed on agar gel electrophoresis and further confirmed by restriction enzyme digestion and sequencing. The complete CDS of $\underline{\sigma} \mathrm{B}$ gene was subcloned in frame into BamHI and HindIII sites of a 6X His-tagged prokaryotic expression vector, pET 32a (+) (Novagen, Germany). The recombinant plasmid (pET32a- $\underline{\sigma} B$ ) was confirmed by restriction enzyme digestion and DNA sequencing.

\section{Expression and purification of recombinant $\underline{\sigma} \mathbf{B}$ protein}

The recombinant plasmid (pET32a- $\underline{-} \mathrm{B}$ ) was used to transform $E$. coli BL21 (DE3) Rosetta cells (Novagen, Germany) and protein production was induced with isopropyl-dthiogalactopyranoside (IPTG; $1 \mathrm{mM}$ final concentration, MBI Fermentas, Germany) for $6 \mathrm{hrs}$ at $37{ }^{\circ} \mathrm{C}$. The bacterial culture pellet containing $6 \mathrm{X}$ Histidine tagged $\underline{\sigma} \mathrm{B}$ protein was solubilised using $8 \mathrm{M}$ urea and purified by affinity chromatography by Ni-NTA column (Invitrogen, USA). The recombinant protein was further purified using the Amicon ultra-4 centrifuge filter device (Millipore, USA) with a membrane cut-off of $30 \mathrm{kDa}$ and dialyzed using $10 \%$ glycerol in PBS. The concentration of purified protein was determined by Modified Lowry's Protein assay kit according to the manufacturer's protocol (Pierce, USA). The confirmation of the recombinant $\underline{\sigma} \mathrm{B}$ protein was accessed by SDS-PAGE and Western blot using anti-His antibody (Qiagen, Germany) as well as hyperimmune sera raised against the recombinant $\underline{\sigma} \mathrm{B}$ protein.

\section{SDS-PAGE and Western blot}

Purified $\underline{\sigma} \mathrm{B}$ protein and protein extracts of the induced recombinant cells were mixed with an equal volume of reducing laemmeli sample buffer, boiled for $3 \mathrm{~min}$, and separated by SDS-PAGE (using 10\% gel, in a BioRad, MiniProtean II electrophoresis unit). After electrophoresis, the fusion proteins and protein extracts of the induced recombinant cells were transferred to nitrocellulose membranes by the method described by Towbin et al., (1979). The blots were incubated overnight at room temperature, with 1:200 dilution of chicken anti-ARV hyper-immune serum to probe the recombinant $\underline{\sigma} \mathrm{B}$ protein.

\section{Results and Discussion}

Reovirus infection is an economically important and highly contagious viral disease of broiler birds which needs timely diagnosis for implementation of effective control measures. Molecular biotechnology-based approaches viz. dot-blot hybridization (Yin and Lee, 1998), PCR combined with RFLP (Hedayati et al., 2013), immunodiffusion and serum neutralization test (Islam and Jones, 1988) has been described for the identification of avian reoviruses in infected chickens. Indirect ELISA kits based on the $\sigma \mathrm{B}$ and $\underline{\sigma} \mathrm{C}$ proteins have been developed for the detection of plasma or serum antibodies against ARV infection in chickens (Liu et al., 2002). Therefore, bulk production of a properly folded, antigenic and immunogenic ARV proteins is an absolute neccesity for 
developent of ELISA based diagnostic kits. Prokaryote based expression system offers the advantage in terms of its simplicity, robustness, low cost and high yield of recombinant proteins over mammalian and yeast based system, which are cumbersome and costly systems (Chen, 2012).

Chicken embryo fibroblast (CEF) cell culture, the most senstive culture system for propagation of $\mathrm{ARV}$, was used in the current study. The cytopathic effects (CPE) were visualized as the formation of multinucleated cells (syncytia), which appeared as early as 24 hr post inoculation, followed by degeneration and detachment from the monolayer leaving small holes in the cell sheath and dead cells floating in the medium (Fig. 1). These CPE observation were in accordance with the previous study in which ARV-infected CEF cells exhibited shrinkage, rounding, and detachment from the plate, nuclear damage, with chromatin condensation and margination (Labrada et al., 2002).

The culture supernatant of CEF, infected with ARV isolate was used for isolation of RNA template which was further employed for amplification of the $\underline{\sigma} \mathrm{B}$ protein encoding gene using gene specific primers. The expected amplicon of $1.1 \mathrm{Kbp}$ was obtained and run on an agarose gel (Fig. 2A). Further, this amplified product was cloned in $\mathrm{pTZ57R/T}$ and the positive colonies were selected by blue-white screening and restriction enzyme digestion that released the $1.1 \mathrm{Kbp} \underline{\sigma} \mathrm{B}$ protein encoding gene insert (Fig. 2B). Recombinant pTZ57R/T- $\underline{\sigma} \mathrm{B}$ clones were subjected to Sanger sequencing and the nucleotide sequence obtained was BLAST analyzed.

Fig.1 Reovirus-induced cytopathic effects observed in chicken embryonic fibroblast cell culture. 1A. Non-infected primary chicken embryo fibroblast cells. 1B. Cytopathic effects observed $48 \mathrm{~h}$ post reovirus infection. Multinucleated cells (syncytia) are indicated by arrow. CPE include degeneration and detachment of fibroblast cells from monolayer leaving small holes in the cell sheath and dead cells floating in the medium
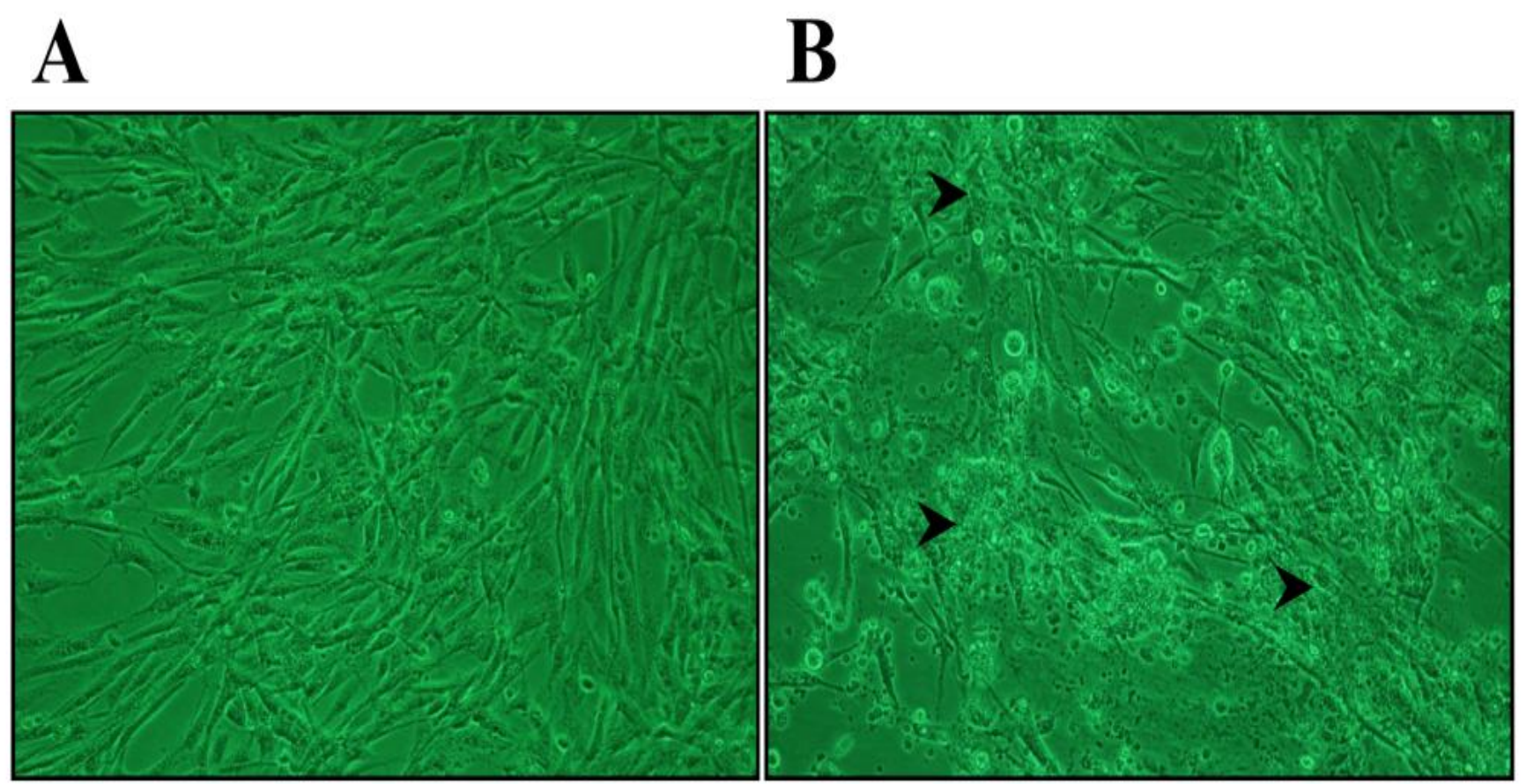
Fig.2 PCR and restriction digestion of $\sigma \mathrm{B}$ gene in cloning and expression vector. 2A. Agarose gel electrophoresis of $\sigma \mathrm{B}$ gene amplicon of $1.1 \mathrm{~Kb}$. Lane 1. $100 \mathrm{bp}$ DNA ladder; lane 2 and 3. $1.1 \mathrm{~Kb} \sigma \mathrm{B}$ gene amplicon. 2B. Agarose gel electrophoresis of BamHI and HindIII digested recombinant pTZ57R/T plasmid confirming the presence of $1.1 \mathrm{~Kb} \sigma \mathrm{B}$ gene amplicon. Lane 1. $1 \mathrm{~Kb}$ DNA ladder. Lane 2. Vector control pTZ57R/T plasmid after single digestion with BamHI; Lane 3 and 4. Recombinant pTZ57R/T plasmid digested with BamHI and HindIII showing the release of $1.1 \mathrm{~Kb} \sigma \mathrm{B}$ gene insert.

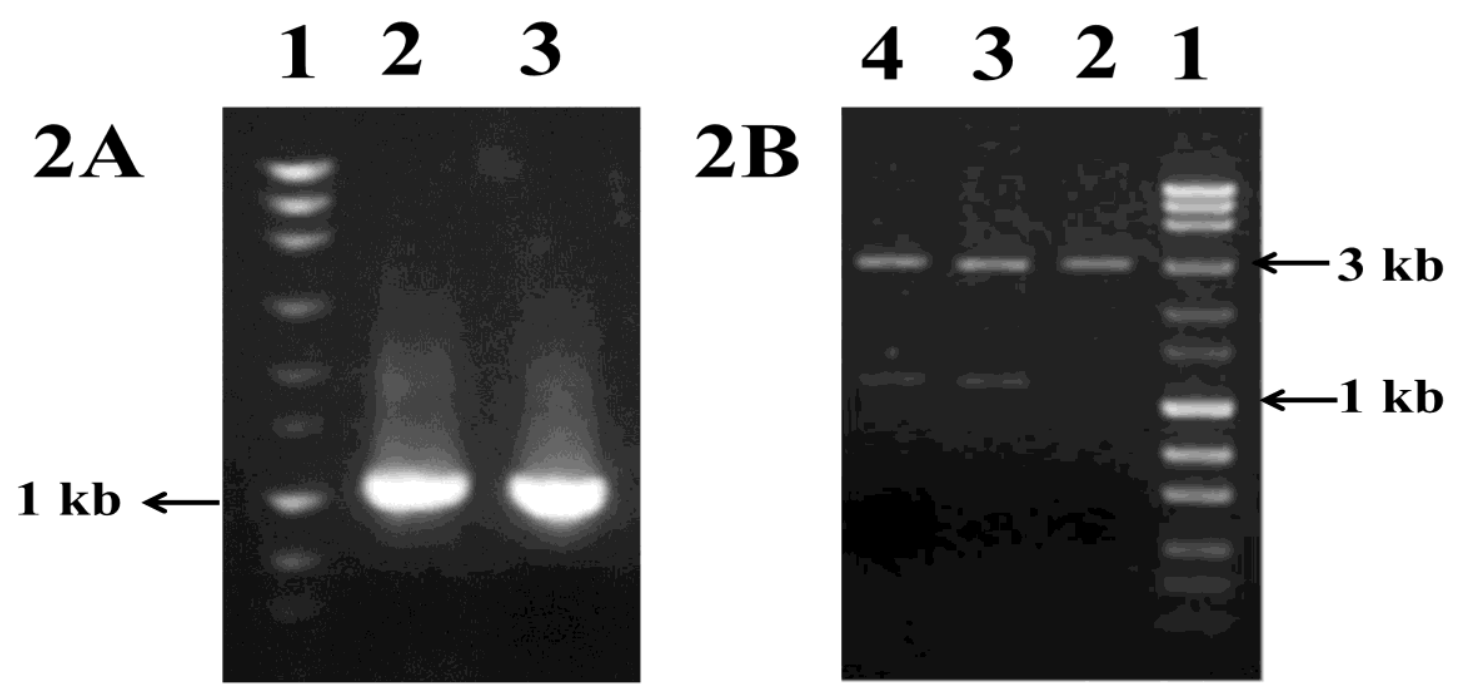

Fig.3 SDS-PAGE and Western blot analysis of $\sigma \mathrm{B}$ expression in a prokaryotic expression system. 3A. SDS-PAGE analysis of $\sigma B$ expression kinetics in BL21 cells. Lane $M$. Pre-stained protein marker; Lane 2. Cell lysate from uninduced cells, lane 2, 3, 4 and 5, Cell lysate from induced cells at 2, 4, 6, and $8 \mathrm{hrs}$ post-induction showing $56 \mathrm{kDa} \sigma \mathrm{B}$ protein in BL21 cells. 3B. Western blot analysis showing $56 \mathrm{kDa} \sigma \mathrm{B}$ protein in BL21 cells. Lane 1 and 2. Cells lysate from post-induction cells showing $56 \mathrm{kDa} \sigma \mathrm{B}$ protein in BL21 cells.

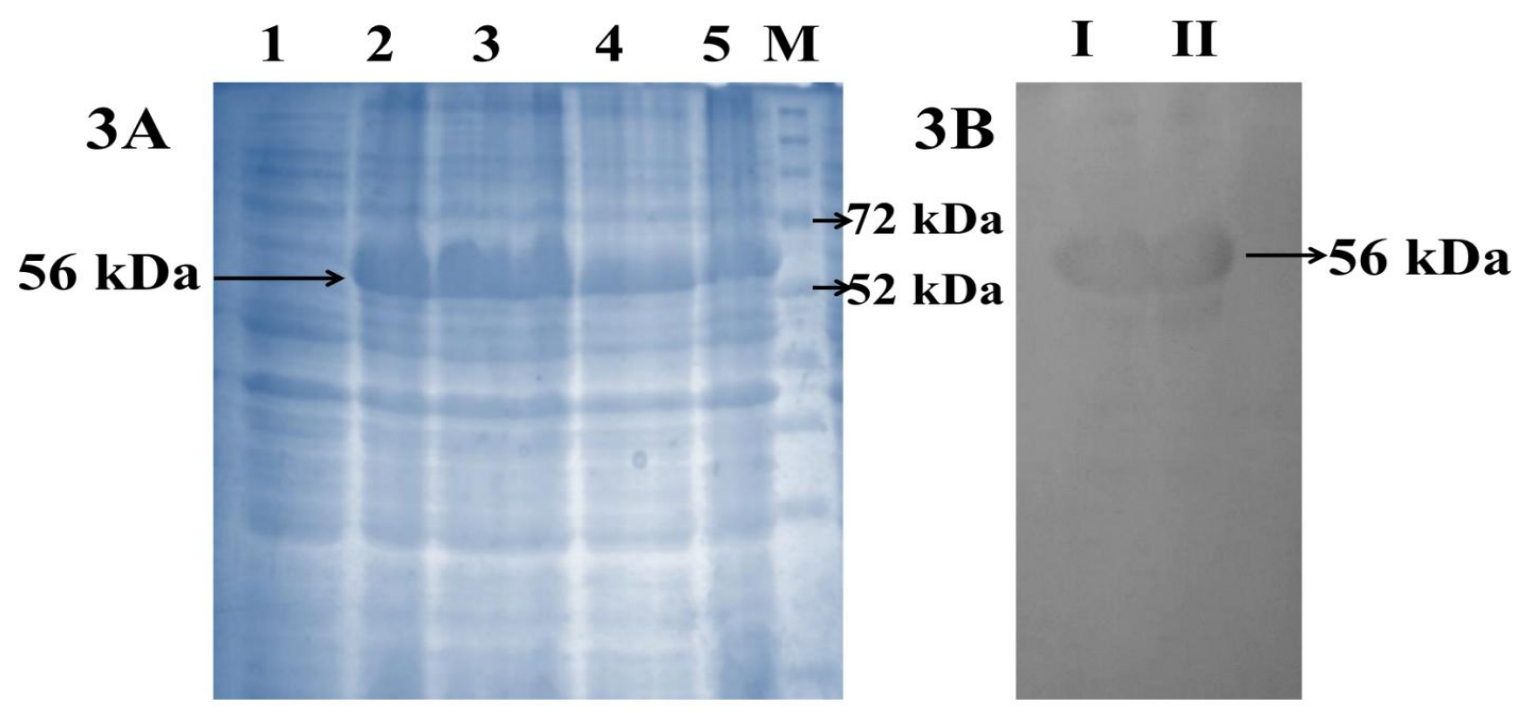


The deduced nucleotide sequence of the $\underline{\sigma} \mathrm{B}$ encoding gene was submitted to GenBank (Acc. No. JQ924284). The nucleotide sequence shared overall $89.9 \%$ sequence homology with ARV isolates from other countries. However, it shared only $64.0 \%$ homology with Muscovy duck reovirus (strain 89026) and 70.6-71.2\% with turkey reovirus (strain NC98 and Tx99, respectively) at nucleotide level (Kumari et al., 2011). This can be attributed to highly variable nature of $\underline{\sigma} \mathrm{B}$ encoding gene as reported earlier (Wickramasinghe et al., 1993).

Further, the amino acid sequence data of $\underline{\sigma} B$ protein of Indian ARV isolate revealed the presence of two conserved B- cell epitopes as well as variable immunogenic epitopes similar to those found in Tunisian isolates (Hellal Kort et al., 2013). These two conserved minimal determinant of linear Bcell epitopes ${ }^{21} \mathrm{KTPACW}^{26}$ (epitope A) and ${ }^{32}$ WDTVTFH $^{38}$ (epitope B) were also identified in the $\sigma B$ protein sequence of American chicken ARV (S1133 strain) isolate (Yin et al., 2013). It has been shown that monoclonal antibodies raised against these epitopes are able to protect birds from infection (Yin at al., 2013). Due to highly variable nature of the $\sigma \mathrm{B}$ protein and its potential role in inducing protective antibodies, there is growing need to develop vaccines and diagnostics according to serotype prevalent in particular area. Therefore, in the present study, expression and purification of $\underline{\sigma} B$ protein of Indian ARV isolate was carried out for further use in the development of an ELISA assay.

E. coli BL21 expression system has been widely used due to various advantages like relative inexpensive cost, ease of manipulation with rapid growth rate (Mayer and Buchner, 2004). Hence in the present study, E. coli BL21 system was choosed for $\underline{\sigma} \mathrm{B}$ protein expression. Following induction with $1 \mathrm{mM}$ IPTG, the recombinant E. coli expressed $\underline{\sigma} \mathrm{B}$ protein along with $\mathrm{N}$-terminus $6 \mathrm{X}$ His-tag accounting for a total molecular weight of $56 \mathrm{kDa}$ as observed on $10 \%$ SDSPAGE (Fig. 3A). The highest expression level was found at $4 \mathrm{hr}$ post-induction as observed on SDS-PAGE. Further, the $\underline{\sigma} \mathrm{B}$ protein was purified under denaturing condition with dialysis and further subjected to SDS-PAGE analysis, which showed a single band of purified recombinant $\underline{\sigma} \mathrm{B}$ protein at the expected size of $56 \mathrm{kDa}$.

In another study, the prokaryotic expression of $\underline{\sigma} \mathrm{B}$ fusion protein from S1133 ARV isolate yielded protein with an approximate molecular mass of $59.3 \mathrm{kDa}$, but the expressed proteins showed varying results when immunoblotted with antiserum from chickens vaccinated with vaccine virus strain S1133 (Liu et al., 2002). Similarly, no antibodies could be detected by ELISA or immunoblot assay in ducks immunized with the recombinant muscovy duck reovirus $\underline{\sigma} \mathrm{B}$ protein and no significant protection was noted after the challenge (Le Gall-Recule et al., 1999). These results has been attributed to the presence of antibodies, specific to different serotypes of ARV prevalent under field conditions, making it unable to get detected by traditional immunoassays based on strain S1133 antigen.

In the present study, the presence of recombinant $\underline{\sigma} B$ protein was confirmed with Western blot analysis using anti-His antibodies and field hyper-immune serum, indicating validation of immunore activity with antibodies against Indian ARV isolates (Fig. 3B). The concentration of purified $\underline{\sigma} B$ protein by modified Lowry's protein assay kit was found to be $1.6 \mathrm{mg} / 100 \mathrm{~mL}$ of bacterial culture.

In conclusion, the results from current study validate the use of prokaryotic expression 
system for abundant production of immunogenic $\underline{\sigma} \mathrm{B}$ protein of ARV. The produced $\underline{\sigma} \mathrm{B}$ protein can be employed in future for the development of in-house ELISA test for rapid and timely detection of ARV outbreaks in India.

\section{References}

Benavente, J., and J. Martínez-Costas, 2007. Avian reovirus: structure and biology. Virus Res., 123: 105-119. doi:10.1016/ j.virusres.2006.09.005

Chen, R., 2012. Bacterial expression systems for recombinant protein production: $\mathrm{E}$. coli and beyond. Biotechnol. Adv., 30: 1102-1107. doi:10.1016/j.biotechadv. 2011.09.013

Hedayati, M., Shojadoost, B., Peighambari, S.M., 2013. Detection of avian reoviruses causing tenosynovitis in breeder flocks in Iran by reverse transcription-polymerase chain reaction (RT-PCR) and restriction enzyme fragment length polymorphism (RFLP). Iran J Vet Med., 7: 135 - 142.

Hellal Kort, Y., Bourogâa, H., Gribaa, L., Scott-Algara, D., Ghram, A., 2013. Molecular characterization of avian reovirus isolates in Tunisia. Virol. J., 10: 12. doi:10.1186/1743-422X-10-12

Ide, P.R., Dewitt, W., 1979. Serological incidence of avian reovirus infection in broiler-breeders and progeny in Nova Scotia. Can. Vet. J. Rev., 20: 348-353.

Islam, M.R., Jones, R.C., 1988. An enzymelinked immunosorbent assay for measuring antibody titre against avian reovirus using a single dilution of serum. Avian Pathol, 17: 411-425. doi:10.1080/03079458808436459

Kataria, J.M., Verma, K.C., Mohanty, G.C., 1982. Studies on viral arthritis in chicken: Isolation and characterization of the causative virus. Indian J. Poultry Sci., 17: 257-261.
Kumari S., Kamble N., Dey S., Madhan Mohan C., Kataria J. M., 2011. Phylogenetic analysis of $\sigma \mathrm{B}$ encoding gene of indigenous avian reovirus isolate. Indian J. Comp. Microbiol. Immunol. Infect. Dis., 32: 12-14.

Labrada, L., Bodelon, G., Vinuela, J., Benavente, J., 2002. Avian Reoviruses Cause Apoptosis in Cultured Cells: Viral Uncoating, but Not Viral Gene Expression, Is Required for Apoptosis Induction. J. Virol., 76: 7932-7941. doi:10.1128/JVI.76.16.7932-7941.2002

Le Gall-Reculé, G., Cherbonnel, M., Arnauld, C., Blanchard, P., Jestin, A., Jestin, V., 1999. Molecular characterization and expression of the S3 gene of muscovy duck reovirus strain 89026. J. Gen. Virol., 80: 195-203. doi:10.1099/00221317-80-1-195

Liu, H.J., Kuo, L.C., Hu, Y.C., Liao, M.H., Lien, Y.Y., 2002. Development of an ELISA for detection of antibodies to avian reovirus in chickens. J. Virol. Methods, 102: 129-138.

Meanger, J., Wickramasinghe, R., Enriquez, C.E., Robertson, M.D., Wilcox, G.E., 1995. Type-specific antigenicity of avian reoviruses. Avian Pathol, 24: 121-134. doi:10.1080/03079459508419053

Ni, Y., Ramig, R.F., 1993. Characterization of Avian Reovirus-Induced Cell Fusion: The Role of Viral Structural Proteins. Virology, 194: 705-714. doi:10.1006/ viro.1993.1311

Towbin, H., Staehelin, T., Gordon, J., 1979. Electrophoretic transfer of proteins from polyacrylamide gels to nitrocellulose sheets: procedure and some applications. Proc. Natl. Acad. Sci. U. S. A., 76: 4350-4354.

Vakharia, V. N., Edwards, G. H., Annadata, M., Simpson, L. H., Mundt, E., 1996. Cloning, sequencing and expression of the S1 and S3 genome segments of 
avian reovirus strain '1733, in: In Proceedings of the International Symposium on Adenovirus and Reovirus Infections in Poultry. Rauschholzhausen, Germany, pp. 168180.

Van Regenmortel, M. H. V., Fauquet, C. M., Bishop, D.H.L., Calisher, C.H., Carsten, E.B., Estes, M.K., Lemon, S.M., Maniloff, J., Mayo, M.A., McGeoch, D.J., Pringle, C.R., Wickner, R.B., 2000. Virus taxonomy: classification and nomenclature of viruses: seventh report of the International Committee on Taxonomy of Viruses. Academic Press, San Diego.

Wickramasinghe, R., Meanger, J., Enriquez, C.E., Wilcox, G.E., 1993. Avian reovirus proteins associated with neutralization of virus infectivity.
Virology, 194: 688-696. doi:10.1006/ viro.1993.1309

Yin, H.S., Lee, L.H., 1998. Development and characterization of a nucleic acid probe for avian reoviruses. Avian Pathol, 27: 423-426.

doi:

10.1080/03079459808419363

Yin, H.S., Shieh, H.K., Lee, L.H., 1997. Characterization of the double-stranded RNA genome segment S3 of avian reovirus. J. Virol. Methods, 67: 93-101.

Zhang, Y., Guo, D., Liu, M., Geng, H., Hu, Q., Liu, Y., Liu, N., 2007. Characterization of the sigmaBencoding genes of muscovy duck reovirus: sigmaC-sigmaB-ELISA for antibodies against duck reovirus in ducks. Vet. Microbiol, 121: 231-241. doi:10.1016/j.vetmic.2006.12.008.

\section{How to cite this article:}

Sanjeevna K. Minhas, Nitin M. Kamble, J.M. Kataria, C. Madhan Mohan and Sohini Dey. 2017. High-Yield Expression and Purification of Recombinant $\sigma \mathrm{B}$ Protein of Avian Reovirus (ARV) in Prokaryotic System. Int.J.Curr.Microbiol.App.Sci. 6(9): 434-441. doi: https://doi.org/10.20546/ijcmas.2017.609.052 